\title{
A Hybrid Feature Selection Method based on IGSBFS and Naïve Bayes for the Diagnosis of Erythemato - Squamous Diseases
}

\author{
S. Aruna \\ Research Scholar \\ Dept of Computer Applications \\ Dr M.G.R Educational and \\ Research Institute University
}

\author{
L. V. Nandakishore \\ Asst Professor \\ Dept of Mathematics \\ Dr M.G.R Educational and \\ Research Institute University
}

\author{
S. P. Rajagopalan \\ Prof Emeritus \\ Dept of Computer Applications \\ Dr M.G.R Educational and \\ Research Institute University
}

\begin{abstract}
In this paper a diagnostic model based on Naive Bayes developed to diagnose erytemato squamous diseases. The hybrid feature selection method, named IGSBFS (Information Gain and Sequential Backward Floating Search), combines the advantages of filters and wrappers to select the optimal feature subset from the original feature set. In IGSBFS, Information Gain acts as filters to remove redundant features and SBFS with Naïve Bayes acts as the wrappers to select the ideal feature subset from the remaining features We conducted experiments in WEKA with 10 fold cross validation. The algorithm selected an optimum feature subset of 10 features with $98.9 \%$ accuracy.
\end{abstract}

\section{General Terms}

Algorithms, Pattern Recognition.

\section{Keywords}

Erythemato squamous diseases, Feature selection, Information Gain, Naïve Bayes, Sequential backward floating search.

\section{INTRODUCTION}

Feature selection plays an important role in dimension reduction for classification [1]. The task of a feature selection algorithm (FSA) offers a computational solution to the problem motivated by a certain definition of relevance. Reduction of feature dimensionality is of much importance in data mining. Feature selection directly reduces the number of original features by selecting a subset of them that still retains enough information for classification. It reduces the dimension and lowers the computation consumption so that it can gain classification performance. The feature selection algorithms designed with different evaluation criteria are divided into two categories: the filter methods and wrappers methods [2]. Filter methods have high computational efficiency than wrapper methods. Wrapper approach achieves better results than filter methods. To combine the advantages of both methods we propose a hybrid algorithm to cut the feature space.

In dermatology the differential diagnosis of erythematosquamous diseases is a difficult problem. The diseases in this group are psoriasis, seboreic dermatitis, lichen planus, pityriasis rosea, chronic dermatitis, and pityriasis rubra pilaris. They have little difference in their clinical and histopathological features. Because of this even with biopsy it is difficult to diagnose the disease. Moreover it may show the features of another disease at the beginning stage and their characteristic features at the following stages.

In this paper we propose a diagnostic model based on Naïve Bayes with hybrid feature selection method to diagnose the eythemato squamous diseases. Remaining of the paper organized as follows. Related work is given in Section 2, Section 3 gives a brief description about the FSA algorithms Information Gain and SBFS, Naïve Bayes algorithm and dermatology dataset used for the experiment. Section 4 discusses the diagnostic model for erythemato squamous diseases, Section 5 gives the results obtained and the concluding remarks are given in Section 6 to address further research issues

\section{RELATED WORK}

There have been several studies reported focusing on the diagnosis of erythemato-squamous diseases using dermatology dataset. Among these studies Guvenir et al[3] conducted the first work on the differential diagnosis of erythemato-squamous diseases. They developed a new classification algorithm, called VFI5 (for Voting Feature Intervals) and applied it to problem of differential diagnosis of Erythemato-Squamous diseases. The VFI5 algorithm achieved $96.2 \%$ accuracy on the Dermatology dataset with 22 histopathological features. Guvenir and Emeksiz [4] presented an expert system for differential diagnosis of erythematosquamous diseases incorporating decision made by three classification algorithms: nearest neighbor classifier, naïve Bayesian classifier and voting feature intervals-5. They obtained $99.2 \%$ classification accuracy on the differential diagnosis of erythemato-squamous diseases. Bojarczuk et al. [5] used a constrained-syntax GP algorithm. The results showed that the proposed GP obtained $96.64 \%$ classification accuracy rate considerably better than $\mathrm{C} 4.5(89.12 \%)$ a very well known advanced decision-tree algorithm often used in machine learning and data mining. Ubeyli and Guler proposed an approach based on adaptive neurofuzzy inference systems for detection of erythemato-squamous diseases [6], and got the classification accuracy of $95.5 \%$.

Luukka and Leppalampi obtained $97.02 \%$ using fuzzy similarity classifier for diagnosis of erythemato-squamous diseases [7]. Polat and Gunes [8] proposed methods based on fuzzy weighted pre-processing, K-NN based weighted preprocessing, and decision tree classifier. Classification accuracy they reached are $88.18 \%, 97.57 \%$, and $99.00 \%$ respectively. Nanni obtained $97.22 \%, 97.22 \%, 97.5 \%, 98.1 \%$, 
97.22\%, 97.5\%, 97.8\%, and 98.3\% using LSVM, RS, B1 5, B1 10, B1 15, B2 5, B2 10, and B2 15 algorithms [9]. Luukka presented similarity classifier using similarity measure derived from Yu's norm for classifying medical data sets [10], and the classification accuracy for the diagnosis of erythemato-squamous diseases was $97.8 \%$. Ubeyli obtained $98.32 \%$ classification accuracy on the differential diagnosis of erythemato-squamous diseases [11], using multiclass support vector machines with the error correcting output codes. Polat and Gunes obtained $96.71 \%$ classification correct rate on diagnosis of erythemato-squamous diseases using a novel hybrid intelligence method based on $\mathrm{C} 4.5$ decision tree classifier and one against all approach for multi-class classification problem [12].

Ubeyli obtained about $97.77 \%$ classification accuracy using combined neural networks model to guide model selection for the diagnosis of erythemato-squamous diseases [13]. Liu et al. obtained $96.72 \%, 92.18 \%, 95.08 \%$, and $92.20 \%$ using feature selection algorithm with dynamic mutual information, estimated using four typical classifiers named Naive Bayes, 1Nearest neighbor, C4.5 and RIPPER [14]. Karabatak and Ince proposed a new feature selection method based on association rules and neural network for diagnosis of erythematosquamous diseases, and their correct classification rate was 98.61\%.[15]. Ubeyli and Dogdu [16] presented an approach based on the implementation of $k$-means clustering for automated detection of erythemato-squamous diseases. They obtained $94.22 \%$ prediction accuracy of the $k$-means clustering. Lekkas and Mikhailov [17] reviewed a method for evolving fuzzy classification which allows data to be processed in online mode by recursively modifying a fuzzy rule base on a per-sample basis from data streams. They obtained the accuracy of $97.55 \%$. Xie and Wang [18] developed a diagnosis model based on support vector machine (SVM) and a novel hybrid feature selection method to diagnose erythemato-squamous diseases. They obtained a classification accuracy of $98.61 \%$.

\section{MATERIALS AND METHODS}

\subsection{Feature Selection Algorithms}

\subsubsection{Information Gain}

Information Gain measures the entropy when the feature is given vs. absent to decide how important a given feature is. Let Attr be the set of all attributes and Ex the set of all training examples, value $(\mathrm{x}, \mathrm{a})$ with $x \in E x$ defines the value of a specific example x for attribute $a \in A t t r$, H specifies the entropy. The information gain for an attribute $a \in A t t r$ is calculated as in equation 1 .

$\mathrm{IG}(\mathrm{Ex}, \mathrm{a})=\mathrm{H}(\mathrm{Ex})-$

$$
\begin{aligned}
& \sum_{\text {vevalues }(a)} \frac{\mid\{n \in E x \mid \text { value }(x, a)=v\} \mid}{|E x|} * \\
& H(\{x \in \operatorname{Ex} \mid \operatorname{value}(x, a)=v\})
\end{aligned}
$$

For each attribute values if a unique classification exists for the result attribute then the information gain is equal to the total entropy where the relative entropy is 0. Equation 2 defines the gain ratio for a test., where $n$ is the number of examples left in the class after the test on the attribute. Equation 3 gives the Information Gain Ratio Calculation.
$\operatorname{IV}(E x, a)=-\sum \frac{|\{n \in E x\}|}{|E x|} * \log _{2}\left(\frac{|\{n \in E x\}|}{|E x|}\right)$

$\operatorname{IGR}(E x, a)=\quad$ IG/IV

\subsubsection{Sequential backward floating search}

Floating search methods [19-20], sequential forward floating selection (SFFS) and sequential backward floating selection (SBFS), are now considered as standard feature selection tools, providing good performance and close-to-optimum or optimum results in most tasks [21-22]. SFBS starts with the full feature set (of size $\mathrm{m}$ ). It do the search until the reach of desired dimension d. The time complexity for SFBS is $\mathrm{O}(\mathrm{m}-$ d). SBFS works best when the optimal feature subset has more number of features, since SBFS spends most of its time visiting large subsets.

\subsubsection{Nä̈ve Bayes}

Naïve Bayes classifier based on Bayes theorem is a probabilistic classifier.It is called as the independent feature model because it considers that all attributes independently contribute to a certain decision. Hence for each class only the variances of the variables determined and not the entire covariance matrix.Taking into account the nature of the underlying probability model, the Naïve Bayes classifier can be trained very efficiently in a supervised learning setting, working better in many complex real-world situations, especially in the computer-aided diagnosis than one might expect [23-24].

\subsubsection{Erythemato squamous data set}

Nilsel Ilter M.D.,Ph.D is the owner of this data set obtained from UCI Machine learning depository (http://archive.ics.uci.edu/ml). This database has 34 attributes, 33 of which are linear valued and one of them is nominal. Patients were first evaluated clinically with 12 features. Then skin samples evaluated for 22 histopathological features by analyzing under a microscope. Number of Instances: 366, Number of Attributes: 34. Table 1, 2, 3 gives the information about the class, clinical and histopathological attributes. Most attributes take values $0,1,2,3$. The 0 value indicates that the clinical or histopathological feature was not present, values 1 and 2 are intermediate for each feature while value 3 means that the feature was the strongest. In case of the family history feature value 0 means that symptoms were not observed in the family, value 1 otherwise.

Table 1. Class distribution

\begin{tabular}{|c|l|c|}
\hline $\begin{array}{c}\text { Class } \\
\text { code }\end{array}$ & \multicolumn{1}{|c|}{ Class Name } & \# of Instances \\
\hline 1 & psoriasis & 112 \\
\hline 2 & seboreic dermatitis & 61 \\
\hline 3 & lichen planus & 72 \\
\hline 4 & pityriasis rosea & 49 \\
\hline 5 & chronic dermatitis & 52 \\
\hline 6 & pityriasis rubra pilaris & 20 \\
\hline
\end{tabular}




\begin{tabular}{|c|c|}
\hline S. No & Attribute name \\
\hline 1 & Erythema \\
\hline 2 & scaling \\
\hline 3 & definite borders \\
\hline 4 & itching \\
\hline 5 & poebner phenomenon \\
\hline 6 & follicular papules \\
\hline 7 & oral mucosal involvement \\
\hline 8 & knee and elbow involvement \\
\hline 9 & scalp involvement \\
\hline 10 & family history, (0 or 1) \\
\hline 11 & Age (linear) \\
\hline 34 &
\end{tabular}

Table 2. Clinical attributes

Table 3. Histo pathological attributes

\begin{tabular}{|c|c|}
\hline S. No & Attribute Name \\
\hline 12 & melanin incontinence \\
\hline 13 & eosinophils in the infiltrate \\
\hline 14 & PNL infiltrate \\
\hline 15 & fibrosis of the papillary dermis \\
\hline 16 & exocytosis \\
\hline 17 & acanthosis \\
\hline 18 & hyperkeratosis \\
\hline 19 & parakeratosis \\
\hline 20 & clubbing of the rete ridges \\
\hline 21 & elongation of the rete ridges \\
\hline 22 & thinning of the suprapapillary epidermis \\
\hline 23 & spongiform pustule \\
\hline 24 & munro microabcess \\
\hline 25 & focal hypergranulosis \\
\hline 26 & disappearance of the granular layer \\
\hline 27 & vacuolisation and damage of basal layer \\
\hline 28 & spongiosis \\
\hline 29 & saw-tooth appearance of retes \\
\hline 30 & follicular horn plug \\
\hline 31 & perifollicular parakeratosis \\
\hline 32 & inflammatory monoluclear inflitrate \\
\hline 33 & band-like infiltrate \\
\hline
\end{tabular}

\section{DIAGNOSTIC MODEL}

In the diagnostic model first the Information Gain method filters the redundant and irrelevant features which results in reduced feature subset. From this subset SBFS based on Naïve bayes searches for the most relevant features resulting in optimum feature set used for diagnosing the disease. Figure 1 explains the stages in the algorithm.

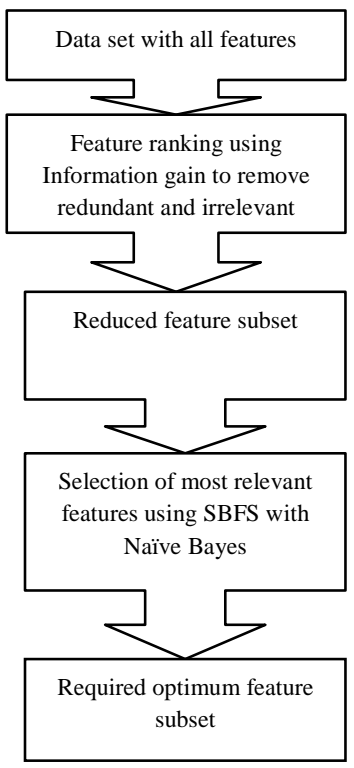

Fig 1. Stages in IGSBFS algorithm

\subsection{IGSBFS algorithm}

This is a greedy algorithm with best first search approach.We rank all the features with Information Gain. After ranking we remove $1 / 3^{\text {rd }}$ features with least gain ratio. With the resultant $2 / 3^{\text {rd }}$ features SBFS with Naïve bayes classifier searches for optimum feature subset. We store the features in a queue in such a way that the features with less gain ratio will be in the front of the queue. Naïve bayes classifier run to get the global optimum. Initially all the features will be in waiting state. When the queue has features in waiting state, a feature in front of the queue is sequentially deleted, kept in a temporary variable and the state of the feature is change to ready state. Naïve bayes classifier run with features in the queue and the accuracy obtained is local optimum. If the local optimum is more than global optimum set the global optimum as the local optimum. If the local optimum is less than the global optimum then backtracking is done i.e. the feature in the temp added to the end of the queue and its state changed to processed state. Otherwise the process continues until the queue has features in waiting state.

\subsubsection{Algorithm}

Input: Data set with all features

Output: Data set with optimum features

Step 1: Rank the features using Information Gain method

Step 2: Remove $1 / 3^{\text {rd }}$ of the features from the feature set which has least score of Gain ratio.

Step 3: Add the remaining $2 / 3^{\text {rd }}$ feature set in a queue in such a way that the front of the queue has features with least Gain ratio. 
Step 4: Set the status of all the features in the queue to waiting state.

Step 5: Run the Nä̈ve Bayes classifier and set the global optimum as accuracy obtained.

Step 6: If the queue has features in waiting state goto step 7 else goto step 12.

Step 7: Move the feature in the front of the queue to temp and change its state as ready

Step 8: Run the Naïve Bayes classifier with features in the queue and the accuracy obtained be local optimum.

Step 9: If the local optimum is more than the global optimum set the global optimum as the current local optimum.

Step 10: If the local optimum is greater than or equal to global optimum delete temp and goto step 6.

Step 11: If the local optimum is less than global optimum change the state of the feature in the temp as processed, add the feature at the end of the queue and goto step 6.

Step 12: The features in the queue is the required optimum feature subset necessary for the diagnostic model.

\section{RESULTS}

Experiments were conducted in WEKA with 10 fold cross validation. Ten fold cross validation has been statistically good enough in evaluating the performance of the classifier [25]. Figure 2 shows the feature ranking using Information gain.

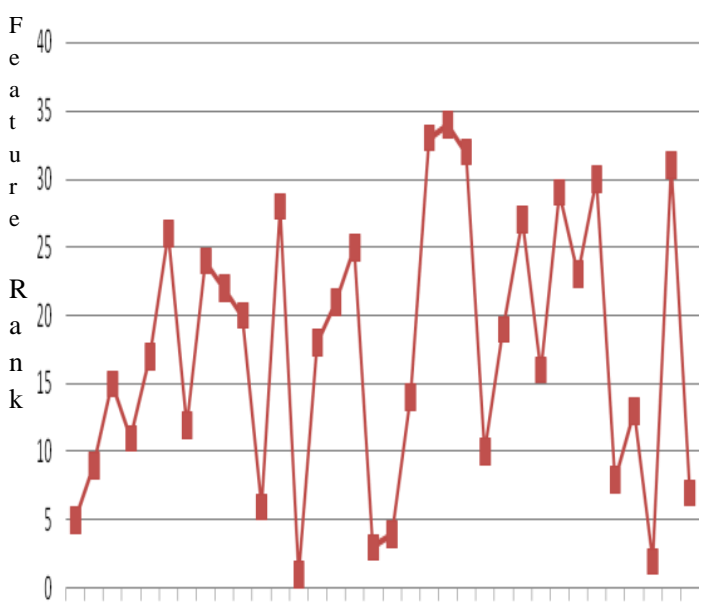

12345678910111213141516171819202122232425262728293031323334

Feature \#

Fig 2. Feature ranking using Information Gain

Algorithm started with 34 features with accuracy of $97.2678 \%$. After ranking with information gain we removed 11 features with least gain ratio namely $13,32,17,18,1,11$, $34,30,2,23,4$. With the resultant 23 features namely 21,20 , $22,33,29,27,12,25,6,16,8,28,9,15,10,24,14,5,26,3$, 19,31 and 7 the accuracy is $98.3607 \%$, Then SBFS method used with Naïve bayes classifier to search for optimum feature subset. This algorithm ended with the accuracy of $98.9071 \%$ with 10 features namely $21,33,6,8,28,15,5,26,31$ and 7 as optimum feature set. The steps carried out in each iteration is shown in Table 4.
Table 4. Steps carried out in each iteration

\begin{tabular}{|c|c|c|c|c|}
\hline \multirow{2}{*}{ Iteration } & \multicolumn{2}{|c|}{ Feature \# } & \multirow{2}{*}{ Accuracy } & \multirow{2}{*}{ Comments } \\
\hline & Removed & Added & & \\
\hline 1 & 7 & & 98.0874 & $\begin{array}{l}\text { L.O<G.O- } \\
\text { Backtrack }\end{array}$ \\
\hline 2 & 31 & 7 & 98.0874 & $\begin{array}{l}\text { L.O<G.O- } \\
\text { Backtrack }\end{array}$ \\
\hline 3 & 19 & 31 & 98.3607 & L.O $=$ G.O \\
\hline 4 & 3 & & 98.6339 & $\begin{array}{l}\text { L.O>G.O- } \\
\text { Update G.O }\end{array}$ \\
\hline 5 & 26 & & 97.8142 & $\begin{array}{l}\text { L.O<G.O- } \\
\text { Backtrack }\end{array}$ \\
\hline 6 & 5 & 26 & 94.2623 & $\begin{array}{l}\text { L.O<G.O- } \\
\text { Backtrack }\end{array}$ \\
\hline 7 & 14 & 5 & 98.9071 & $\begin{array}{l}\text { L.O>G.O- } \\
\text { Update G.O }\end{array}$ \\
\hline 8 & 24 & & 98.9071 & L.O=G.O \\
\hline 9 & 10 & & 98.9071 & L.O $=$ G.O \\
\hline 10 & 15 & & 96.1749 & $\begin{array}{l}\text { L.O<G.O- } \\
\text { Backtrack }\end{array}$ \\
\hline 11 & 9 & 15 & 98.9071 & L.O=G.O \\
\hline 12 & 28 & & 98.6339 & $\begin{array}{l}\text { L.O<G.O- } \\
\text { Backtrack }\end{array}$ \\
\hline 13 & 8 & 28 & 98.6339 & $\begin{array}{l}\text { L.O }<\text { G.O- } \\
\text { Backtrack }\end{array}$ \\
\hline 14 & 16 & 8 & 98.9071 & L.O=G.O \\
\hline 15 & 6 & & 98.6339 & $\begin{array}{l}\text { L.O<G.O- } \\
\text { Backtrack }\end{array}$ \\
\hline 16 & 25 & 6 & 98.9071 & L. $\mathrm{O}=\mathrm{G} . \mathrm{O}$ \\
\hline 17 & 12 & & 98.9071 & L.O=G.O \\
\hline 18 & 27 & & 98.9071 & L.O=G.O \\
\hline 19 & 29 & & 98.9071 & L.O=G.O \\
\hline 20 & 33 & & 98.0874 & $\begin{array}{l}\text { L.O<G.O- } \\
\text { Backtrack }\end{array}$ \\
\hline 21 & 22 & 33 & 98.9071 & L.O=G.O \\
\hline 22 & 20 & & 98.9071 & L.O $=$ G.O \\
\hline 23 & 21 & & 97.2678 & $\begin{array}{l}\text { L.O<G.O- } \\
\text { Backtrack }\end{array}$ \\
\hline
\end{tabular}

We used the data mining algorithms such as Decision tree (Simple Cart), RBF Network, SMO RBF Kernel and Poly Kernel to classify the erythemato squamous dataset with all features and with optimum features selected by our hybrid algorithm. Table 5 and 6 shows the results for the classifiers. The empirical comparison shows that the features selected by the hybrid algorithm also improved the accuracy of all the data mining algorithms. Table 7 shows the classification accuracies of our method and other classifiers from literature for the dermatology data set. 
Table 5. Accuracy, mean absolute and root mean squared error for various datamining algorithms without feature selection

\begin{tabular}{|c|c|c|c|}
\hline Algorithm & Accuracy & $\begin{array}{c}\text { Mean absolute } \\
\text { error }\end{array}$ & $\begin{array}{c}\text { Root mean } \\
\text { squared } \\
\text { error }\end{array}$ \\
\hline Simple Cart & 93.9891 & 0.0267 & 0.1401 \\
\hline $\begin{array}{c}\text { RBF } \\
\text { Network }\end{array}$ & 96.1749 & 0.0132 & 0.1112 \\
\hline $\begin{array}{c}\text { RBF } \\
\text { Kernel(c-10, } \\
\text { G-0.1) }\end{array}$ & 95.9016 & 0.2231 & 0.3117 \\
\hline $\begin{array}{c}\text { SMO-Poly } \\
\text { Kernel( E- } \\
1.0)\end{array}$ & 95.3552 & 0.2233 & 0.3119 \\
\hline
\end{tabular}

Table 6. Accuracy, mean absolute and root mean squared error for various datamining algorithms with 10 features selected by our algorithm

\begin{tabular}{|c|c|c|c|}
\hline Algorithm & Accuracy & $\begin{array}{c}\text { Mean absolute } \\
\text { error }\end{array}$ & $\begin{array}{c}\text { Root mean } \\
\text { squared } \\
\text { error }\end{array}$ \\
\hline Simple Cart & 95.6284 & 0.0217 & 0.1189 \\
\hline $\begin{array}{c}\text { RBF } \\
\text { Network }\end{array}$ & 97.2678 & 0.0117 & 0.0959 \\
\hline $\begin{array}{c}\text { RBF } \\
\text { Kernel(c-10, } \\
\text { G-0.1) }\end{array}$ & 98.0874 & 0.2228 & 0.3107 \\
\hline $\begin{array}{c}\text { SMO-Poly } \\
\text { Kernel( E- } \\
1.0)\end{array}$ & 98.3607 & 0.2226 & 0.3103 \\
\hline
\end{tabular}

Table 7. Classification accuracies of our method and other classifiers from literature

\begin{tabular}{|c|c|c|c|}
\hline Author[Ref] & Year & Method & $\operatorname{Accuracy}(\%)$ \\
\hline $\begin{array}{l}\text { Guvenir et al. } \\
\text { [3] }\end{array}$ & 1998 & VFI5 & 96.2 \\
\hline $\begin{array}{l}\text { Guvenir and } \\
\text { Emeksiz [4] }\end{array}$ & 2000 & $\begin{array}{l}\text { Nearest } \\
\text { neighbor } \\
\text { classifier } \\
\text { Naïve Bayesian } \\
\text { classifier } \\
\text { VFI5 }\end{array}$ & 99.2 \\
\hline Bojarczuk [5] & 2001 & $\begin{array}{l}\text { A constrained- } \\
\text { syntax genetic } \\
\text { programming } \\
\text { C4.5 }\end{array}$ & $\begin{array}{l}96.64 \\
89.12\end{array}$ \\
\hline $\begin{array}{l}\text { Ubeyli and } \\
\text { Guler [6] }\end{array}$ & 2005 & ANFIS & 95.50 \\
\hline $\begin{array}{l}\text { Luukka and } \\
\text { Leppalampi } \\
\text { [7] }\end{array}$ & 2006 & $\begin{array}{l}\text { Fuzzy } \\
\text { similarity-based } \\
\text { classification }\end{array}$ & 97.02 \\
\hline $\begin{array}{l}\text { Polat and } \\
\text { Gunes [8] }\end{array}$ & 2006 & $\begin{array}{l}\text { Fuzzy weighted } \\
\text { pre processing } \\
\text { K-NN based } \\
\text { weighted pre- } \\
\text { processing } \\
\text { Decision tree }\end{array}$ & $\begin{array}{l}88.18 \\
97.57 \\
99.00\end{array}$ \\
\hline
\end{tabular}

\begin{tabular}{|l|c|l|c|}
\hline Nani [9] & 2006 & LSVM & 97.22 \\
& & RS & 97.22 \\
& & B1_5 & 97.50 \\
& & B1_10 & 98.10 \\
& & B1_15 & 97.22 \\
& & B2_5 & 97.50 \\
& & B2_10 & 97.80 \\
& & B2_15 & 98.30 \\
\hline Luukka [10] & 2007 & Similarity & 97.80 \\
& & measure & \\
\hline Ubeyli[11] & 2008 & Multiclass SVM & 98.32 \\
\hline $\begin{array}{l}\text { Polat and } \\
\text { Gunes [12] }\end{array}$ & 2009 & With the ECOC & \\
\hline Ubeyli [13] & 2009 & against-all & 96.71 \\
\hline Liu et al. [14] & 2009 & Naive Bayes & 96.72 \\
& & 1-NN & 92.18 \\
& & C4.5 & 95.08 \\
\hline $\begin{array}{l}\text { Karabatak } \\
\text { and Ince [15] }\end{array}$ & 2009 & PIPPER & 92.20 \\
\hline $\begin{array}{l}\text { Ubeyli and } \\
\text { Dogdu [16] }\end{array}$ & 2010 & KR and NN & 98.61 \\
\hline $\begin{array}{l}\text { Lekkas and } \\
\text { Mikhailov } \\
\text { [17] }\end{array}$ & 2010 & Evolustering & 94.22 \\
\hline $\begin{array}{l}\text { Xie and } \\
\text { Wang [18] }\end{array}$ & 2011 & IFSFS fuzzy & 97.55 \\
\hline This study (10 features) & IGSBFS with & 98.90 \\
\hline
\end{tabular}

\section{CONCLUSION}

Feature selection, as a preprocessing step to machine learning especially in real world data, has been very effective in reducing dimensionality, removing irrelevant data, and noise from data to improving result comprehensibility. In this study a hybrid feature selection method based on Naïve bayes proposed for the diagnosis of erythemato squamous disease. It combines the advantages of filters and wrappers. Information gain ratio computed for the features act as evaluation criteria of filters to remove redundant and irrelevant features. SBFS with Naïve bayes acts as wrappers to select the optimum feature subset. Experiment results show that the new hybrid feature selection method obtained optimal classification accuracy with 10 features from a set of 34 features. The optimal feature subset thus obtained then trained with various data mining algorithms such as decision tree (CART), RBF Neural networks, SMO- Poly kernel and RBF kernel to diagnose erythemato squamous diseases. Experimental results show that classification accuracies improved for all data mining algorithms. We conducted the experiments in WEKA with 10 fold cross validation. In this study we used dermatology data set. We use this algorithm in future with various datasets and various combinations of feature selection algorithms will also be considered to select the optimal feature subset. 


\section{REFERENCES}

[1] Y. liu and Y. F. Zheng, (2006) "FS_SFS: A novel feature selection method for support vector machines", Pattern Recognition, vol. 39, pp. 1333-1345.

[2] L. Talavera,(2005) "An evaluation of filter and wrapper methods for feature selection in categorical clustering" Proceedings of 6th international symposium on intelligent data analysis, Madrid, Spain, pp. 440-445.

[3] H. A. Govenir, G. Demiroz, and N. Ilter,( 1998) "Learning differential diagnosis of Eryhemato-Squamous diseases using voting feature intervals", Artificial Intelligence in Medicine, vol. 13, pp. 147-165.

[4] H.A. Guvenir and N. Emeksiz,( 2000) "An expert system for the differential diagnosis of erythemato-squamous diseases", Expert Systems with Applications, vol. 18, pp. 43-49.

[5] Bojarczuk , C. C., Lopes, H. S., Freitas, A. A., ,( 2001) "Data Mining with Constrained-Syntax Genetic Programming: Applications in Medical Data Set", Data Analysis in Medicine and Pharmacology (IDAMAP2001), a Workshop at Medinfo-2001, London, UK.

[6] E. D. Ubeyli and I. Guler,(2005) "Automatic detection of erythemato-squamous diseases usingadaptive neurofuzzy inference systems", Computers in Biology and Medicine, 35:421-433.

[7] P. Luukka and T. Leppalampi.( 2006) "Similarity classifier with generalized mean applied to medical data", Computers in Biology and Medicine, 36:10261040 .

[8] K. Polat and S. Gunes.( 2006) "The effect to diagnostic accuracy of decision tree classifier of fuzzyand k-nn based weighted pre-processing methods to diagnosis of erythemato-squamousdiseases", Digital Signal Processing, 16:922-930.

[9] L. Nanni.( 2006) "An ensemble of classifiers for the diagnosis of erythemato-squamous diseases", Neurocomputing, 69:842-845.

[10] P. Luukka,( 2007) "Similarity classifier using similarity measure derived from yu's norms in classification of medical data sets", Computers in Biology and Medicine, $37: 1133-1140$

[11] E. D. Ubeyli,( 2008) "Multiclass support vector machines for diagnosis of erythemato-squamous diseases", Expert Systems with Applications, 35:17331740 .

[12] K. Polat and S. Gunes,( 2009) "A novel hybrid intelligent method based on $\mathrm{C} 4.5$ decision tree classifier and oneagainst-all approach for multi-class classification problems", Expert Systems with Applications, vol. 36, no. 2, pp. 1587-1592.

[13] E. D. Ubeyli,( 2009) "Combined neural networks for diagnosis of erythemato-squamous diseases", Expert Systems with Applications, 36:5107-5112.

[14] H. W. Liu, J.G. Sun, L. Liu, and H. J. Zhang.( 2009) "Feature selection with dynamic mutual information", Pattern Recognition, 42:1330-1339.

[15] M. Karabatak and M. C. Ince,( 2009) "A new feature selection method based on association rulesfor diagnosis of erythemato-squamous diseases", Expert Systems with Applications, 36: 12500-12505.

[16] E. D. Ubeyli and E. Dogdu,( 2010) "Automatic Detection of Erythemato-Squamous Diseases Using k-Means Clustering", Journal of Medical Systems, vol. 34, pp. 179-184.

[17] Stavros Lekkas and Ludmil Mikhailov,(2010) "Evolving fuzzy medical diagnosis of Pima Indians diabetes and of dermatologica diseases," Artificial Intelligence in Medicine, vol. 50, pp. 117-126.

[18] J. Xie and Ch. Wang,( 2011) "Using support vector machines with a novel hybrid feature selection method for diagnosis of erythemato-squamous diseases", Expert Systems with Applications, vol. 38, no. 5, pp. 5809-5815.

[19] P. Pudil , J. Novovicova, J. Kittler, (1994) "Floating Search Methods in Feature Selection", Pattern Recognition Letters 15 1119-1125.

[20] P. Pudil, J. Novovicova, P. Somol,(2003) "Recent Feature Selection Methods in Statistical Pattern Recognition”, Pattern Recognition and String Matching, Springer-Verlag, Berlin Heidelberg New York.

[21] A.K. Jain, D. Zongker,(1997) "Feature selection: evaluation, application and small sample performance", IEEE Trans. PAMI 19 153-158.

[22] M. Kudo, J. Sklansky,(2000) "Comparison of algorithms that select features for pattern classifiers", Pattern Recognition 33 25-41

[23] S. Belciug, (2008) "Bayesian classification vs. k-nearest neighbour classification for the non-invasive hepatic cancer detection", Proc. 8th International conference on Artificial Intelligence and Digital Communications.

[24] F. Gorunescu, (2006) Data Mining: Concepts, models and techniques, Blue Publishing House, Cluj Napoca.

[25] P. Baldi, S. Brunak, Y. Chauvin, et al. (2000) "Assessing the accuracy of prediction algorithms for classification and overview", Bioinformatics, 5(5):412-424. 